\title{
Evaluating the Role of Vision and Force Feedback in Minimally Invasive Surgery: New Automated Laparoscopic Grasper and a Case Study ${ }^{1}$
}

\author{
Gregory Tholey ${ }^{1}$, Jaydev P. Desai ${ }^{1}$, and Andres E. Castellanos ${ }^{2}$ \\ ${ }^{1}$ Program for Robotics, Intelligent Sensing, and Mechatronics (PRISM) Laboratory \\ 3141 Chestnut Street, MEM Department, Room 2-115 \\ Drexel University, Philadelphia, PA 19104, USA \\ ${ }^{2}$ Department of Surgery, Drexel University College of Medicine, Philadelphia, \\ PA 19104, USA \\ \{gtholey, desai\}@coe.drexel.edu, andres.e.castellanos@drexel.edu
}

\begin{abstract}
Conventional laparoscopic tools do not have the ability of providing force feedback to a surgeon during surgical procedures. Loss of haptic feedback in MIS procedures is a disadvantage to surgeons since they are conventionally used to palpating tissues to diagnose whether the tissue is normal or abnormal. Therefore, a need exists to incorporate force feedback into laparoscopic tools. We have developed a laparoscopic grasper with force feedback capability to help surgeons characterize tissues. Through our interface, the surgeon can feel the tissue grasping forces while interacting with the PHANToM (haptic interface device). Our laparoscopic tool has a modular design for easy conversion between a grasper, cutter, and dissector. We have done several experiments to test two hypotheses with human subjects (20 subjects; 10 surgeons and 10 non-surgeons) to characterize tissues of varying hardness using only vision, only force, and simultaneous vision and force feedback.
\end{abstract}

\section{Introduction}

Minimally invasive surgical (MIS) procedures using long instruments have profoundly influenced modern surgery by decreasing invasiveness, therefore minimizing patient recovery time and cost. However, surgical procedures using long tools inserted through small ports on the body deprive surgeons of the depth perception, dexterity, sense of touch, and straightforward hand eye coordination that they are accustomed to in open procedures and use to diagnose tissue as normal or abnormal [1]. In addition, current robotic surgical systems lack force feedback capabilities as the surgeon is indirectly in contact with the surgical site via the robot. Therefore, the need to incorporate force feedback capabilities into MIS procedures, especially robotically assisted procedures, provides an excellent opportunity to improve the quality of surgical procedures. Improvements in MIS systems will lead to significant societal impacts

\footnotetext{
${ }^{1}$ We would like to acknowledge the support of National Science Foundation grants: EIA0079830, and CAREER Award IIS-0133471 for this work.
} 
through better patient care, reduced morbidity, shorter hospital stays, reduced trauma, faster recovery times, and lower health care costs.

Several researchers have already proposed solutions to incorporate force feedback into current laparoscopic tools through the addition of strains gages and developing new robotic manipulators or laparoscopic tools with incorporated force feedback [28]. However, there still exist many problems within the designs of laparoscopic tools and their use in robotic surgery $[9,10]$. Currently, most experimental force feedback graspers lack friction analysis in their mechanism. Additionally, many of the current tools used in MIS have significant backlash and a large "play" within the linkage that operates the end effector (grasper, cutter, dissector). These problems inadvertently lead to inaccurate positioning of the end effector jaws based on the movement of the handle. As a result of these shortcomings, our goal was to design and develop a modular laparoscopic tool, which would provide force feedback for tissue characterization. Our design addresses these challenges and we have done friction modeling over the range of motion to provide a nearly "transparent" user interface with force feedback for the surgeon.

\section{Development and Modeling}

Our design for the laparoscopic grasper with force feedback capability was guided by constraints associated with current laparoscopic tools. Conventional laparoscopic tools have long, slender tubes that are usually less than $15 \mathrm{~mm}$ in diameter to allow for small incisions. As a result, our first constraint was to restrict the outer diameter of the tube to $15 \mathrm{~mm}$. The next constraint was limiting the movement of the end effector to at most 90 degrees as in conventional laparoscopic graspers. This allows the surgeon to grasp, cut or dissect very thick tissue. Finally, we must achieve accurate positioning of jaws in relation to the handle of the tool through a transmission with minimal friction and nearly zero backlash.

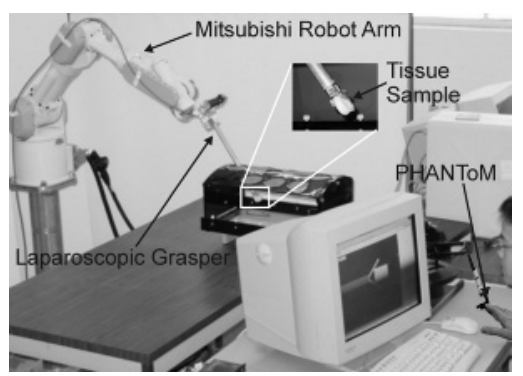

Fig. 1. The laparoscopic grasper attached to the Mitsubishi robot arm for tissue manipulation

torque from the motor to the jaws.

Our initial prototype (see figure 2) has been designed as a laparoscopic grasper; therefore the end effector consists of two serrated jaws (see figure 3) to facilitate grasping. In addition, the jaws have also been designed with a quick-change feature. 
Therefore, we can interchange the jaws to convert the tool to a dissector or cutter. We designed this capability as our concurrent research in the laboratory is focused on reality-based modeling for tissue interaction forces in grasping, cutting, and dissection tasks in MIS.

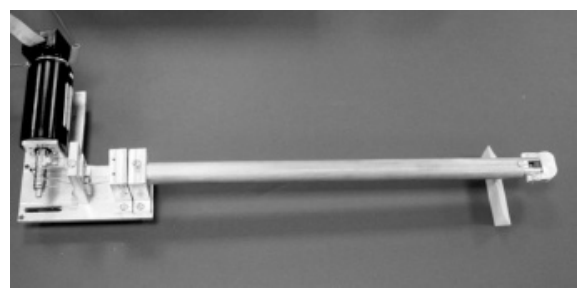

Fig. 2. Prototype of the laparoscopic grasper with force feedback.

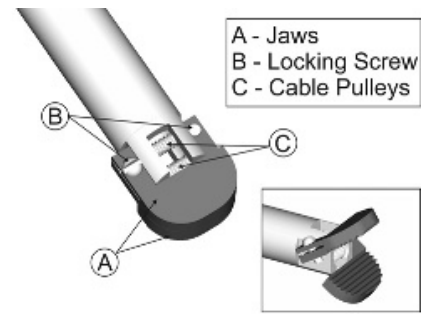

Fig. 3. Lower jaw assembly.

The kinematics of this mechanism are straightforward and involve only the motor pulley and an end effector pulley with a reduction ratio of 1:2.71. The resulting equation relating the end effector force $\mathrm{F}_{\mathrm{j}}$ to the motor torque $\mathrm{T}_{\mathrm{m}}$ is given by:

$$
F_{j}=19.3 * T_{m} \text { (In the absence of friction) . }
$$

The system comprises of the laparoscopic grasper actuated by a DC motor (model RE36, manufactured by Maxon) and the dSpace DS1103 controller board (manufactured by dSPACE, GmBH). We have developed a program that allows the user to input the desired position and as the grasper jaws move, force feedback is provided to the user in real-time through the PHANToM (manufactured by Sensable Technologies, Inc.). Through appropriate calibration, the end effector forces are obtained from the motor current. We implemented a PD controller to control the position of the jaws. The control law is given by:

$$
\mathrm{T}=K_{p}\left(q_{d}-q\right)+K_{v}\left(\dot{q}_{d}-\dot{q}\right)
$$

where $\mathrm{T}$ is the motor torque, $\mathrm{K}_{\mathrm{p}}$ and $\mathrm{K}_{\mathrm{v}}$ are the proportional and integral gains, $q_{d}$ and $q$ are the desired and actual positions, and $\dot{q}_{d}$ and $\dot{q}$ are the desired and actual velocities.

We also measured the friction present in the mechanism by opening/closing the jaws at a very small velocity $(0.77 \mathrm{deg} / \mathrm{s})$. This friction was then fed forwarded into the controller, giving us the modified control law:

$$
\mathrm{T}=K_{p}\left(q_{d}-q\right)+K_{v}\left(\dot{q}_{d}-\dot{q}\right)+\tau_{f r} .
$$

where $\tau_{\mathrm{fr}}$ is the friction torque.

\section{Experiments}

\subsection{Experimental Setup and Research Protocol}

Finally, we were interested in evaluating the role of only vision feedback, only force feedback, simultaneous vision and force feedback and direct exploration in tissue 
characterization. For this, we developed several artificial tissue samples made up of Hydrogel material. The Hydrogel is created using a combination of polyvinyl alcohol (PVA) and polyvinyl pyrrolidone (PVP) in the consistency of 90\% PVA and 10\% PVP. This solution was then caste into molds and subjected to several freezing/thawing cycles. Six cycles were performed and after each cycle a sample was removed. The tissues were numbered from 1 to 6 (in the order they were removed) with sample 1 being the softest tissue while sample 6 was the hardest tissue.

The first test consisted of using only vision to rank 3 tissue samples of varying stiffness from softest to hardest. One sample at a time was grasped with the jaws closing on the tissue to a desired angle. The subject was able to view the sample through a video screen from a standard CCD video camera (using an isometric view of the tissue) in order to view the deformation of the sample. Once the 3 samples (soft, medium, hard) were presented, the subject was asked to rank them from softest to hardest based on their observations. It should be noted that during each trial the subject could request a replay of one or more of the samples before ranking the samples, therefore reducing error due to random guessing or forgetting. The process was repeated for a total of five trials with the samples randomly arrayed for each trial.

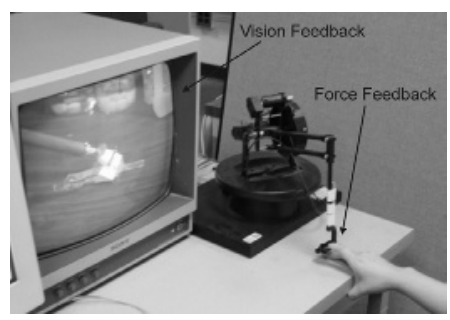

Fig. 4. Subject interaction with PHANToM to obtain force feedback from the grasper.

The second test consisted of the same method with the exception of using force feedback instead of vision feedback to characterize the tissue. For this experiment, the subject interacted with the PHANToM by inserting their finger in a thimble attached to the PHANToM (See figure 4). The direction of the force feedback within the PHANToM was vertical and acting upwards. Therefore, the subject was required to hold the edge of the desk with their thumb under the desk and other three fingers resting on top of the desk. This setup was representative of palpation done by surgeons where arm dynamics do not affect the results. Through the keyboard, an operator opened/closed the jaws while the subject received the force feedback from each sample. As in the first test, the grasper was set to close the jaws to the same angle for each sample in order to keep the deformation constant. The subject could manipulate the PHANToM with their index finger to characterize the amount of grasping force for that particular sample. Once the three samples were presented, the subject was asked to rank the samples in the order from softest to hardest. This process was also repeated for a total of five trials.

The third test consisted of using both force feedback and vision feedback to differentiate between the three tissue samples. This test was performed in the same way as the force test with the exception of having video feedback of the grasping task. The final test of this experiment was to rank the three tissue samples softest to hardest based on direct exploration of the samples with their fingers.

\subsection{Data Collection and Analysis}

The experiment was performed by 20 subjects for a total of 900 trials ( 5 trials for $\mathrm{V}$, $\mathrm{F}, \mathrm{V}+\mathrm{F}$ and 3 tissue samples to be characterized; hence 45 trials by each subject); 10 of which were surgeons who had experience in minimally invasive surgery and 10 of 
which were non-surgeons who had no surgical experience. The data was collected in a qualitative fashion with responses characterized as either "true" or "false" and denoted by a value of 1 or 0 respectively. For a particular trial to be characterized as true, the subject must have identified all three tissue samples in their correct order of stiffness. The data was then analyzed using a single factor analysis of variance (ANOVA) method. The ANOVA method tests two or more data sets to determine if there is a significant statistical difference between the data sets. ANOVA generates a p-value (probability) for the null hypothesis $\left(\mathrm{H}_{0}\right)$ and thus a probability for the hypothesis $\left(\mathrm{H}_{1}\right)$ to be tested. The lower the p-value, the smaller the probability for the null hypothesis to be true and consequently higher is the probability that there is a significant statistical difference between the data sets (or the research hypothesis $\mathrm{H}_{1}$ to be true). The level of significance (alpha value) that we used was 0.05 ; meaning that a hypothesis would be considered true if the p-value was less than the alpha value $(p<\alpha)$. In addition, Tukey's method was used as the post hoc ANOVA test. Tukey's method is used to test all pairwise mean comparisons after an analysis of variance in which the null hypothesis is rejected. This method compares the difference in the means between the data sets with a pre-determined number that corresponds to the level of significance $(\alpha)$. The pre-determined number is obtained from the following equation:

$$
\mathrm{T}_{\alpha}=q_{\alpha}(a, f) \sqrt{\frac{M S E}{n}} .
$$

where $\alpha=$ level of significance $(0.05), a=$ number of populations, $f=$ number of samples taken - number of populations, MSE = mean square error, and $n=$ number of samples per population. Therefore, when $\mathrm{T}_{\alpha}$ is compared to the difference in the means of two data sets, a value greater than $\mathrm{T}_{\alpha}$ would signify a level of significance of the null hypothesis less than $\alpha$ and hence a statistically significant difference between the data sets.

\subsection{Research Hypotheses}

We performed the above experiments to test the validity of two research hypotheses that have direct clinical relevance. These are:

1. Providing only force feedback leads to better tissue characterization for all three samples compared to using only vision feedback for all three samples $\left(\mathrm{F}_{\mathrm{a}}>\mathrm{V}_{\mathrm{a}}\right)$.

2. Providing simultaneous vision and force feedback for all three samples is better than only vision feedback or only force feedback for all three samples $\left((\mathrm{V}+\mathrm{F})_{\mathrm{a}}>\mathrm{V}_{\mathrm{a}},(\mathrm{V}+\mathrm{F})_{\mathrm{a}}>\mathrm{F}_{\mathrm{a}}\right)$.

The ">" sign denotes "is better than" in the above hypotheses. Subscripts "s", "h", and "a" refer to soft tissue, hard tissue, and all three tissues respectively. Also, we will denote only vision feedback, only force feedback, simultaneous vision and force feedback, and direct exploration by $\mathrm{V}, \mathrm{F}, \mathrm{V}+\mathrm{F}$, and DE respectively. 


\subsection{Experimental Results}

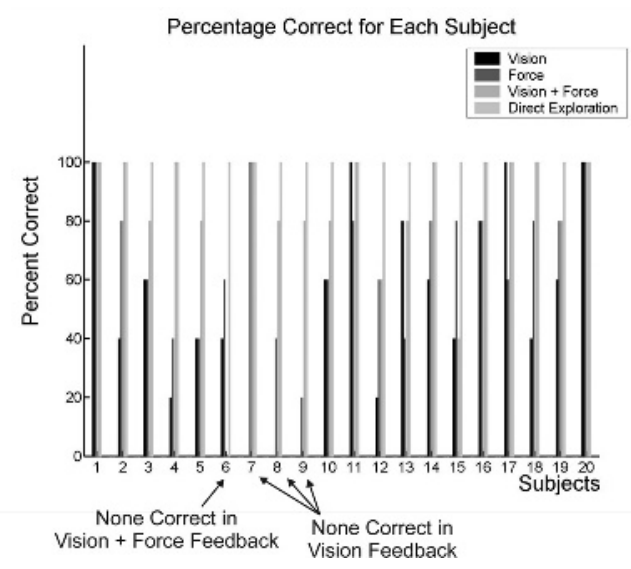

Fig. 5. Percentage of successful tissue characteriziation for each individual using each of the four methods
The individual results for each subject in each of the four methods are shown is figure 5. Each individual performed five trials in each of the methods except direct exploration. The subject needed only to distinguish between the samples using direct exploration once because of the relative easiness of this method compared to the others. Overall, the average correct response using only vision feedback was $52 \%$ and using only force feedback was $67 \%$. Providing both vision and force produced an average of $83 \%$ and direct exploration produced an average of $100 \%$ correct. An ANOVA analysis was performed to evaluate

whether there was a significant difference between the data sets and generated a $\mathrm{p}$ value of 0.00001325 , leading to a probability of greater than $99.99 \%$ that there was a significant statistical difference between the data sets.

Hypothesis 1: Providing only force feedback leads to better tissue characterization for all three samples compared to using only vision feedback for all three samples $\left(F_{a}>V_{a}\right)$.

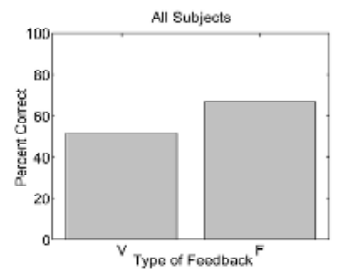

Fig. 6. Percent correct tissue characterizations between the three different artificial tissues using only vision and only force feedback.

Our first hypothesis states that providing force feedback alone would lead to better tissue characterization compared to vision feedback alone. As shown in figure 6, providing vision feedback alone and force feedback alone resulted in differentiating the tissue samples correctly in $50 \%$ and $67 \%$ of the trials respectively. In addition, Tukey's method was used to determine the statistical significance between the data sets consisting of only vision feedback and only force feedback. Using a level of significance of 0.05 , the value of $\mathrm{T}_{0.05}$ was calculated to be 0.1525 . Comparing this to the difference between the means $(0.15)$ of the data sets, the $T_{0.05}$ value is greater and therefore we cannot declare a significant statistical difference. However, the two values are very close and would indicate a probability of the null hypothesis of $5.2 \%$.

Individually, surgeons were correct in $62 \%$ and $72 \%$ of the trials for only vision feedback and only force feedback respectively while non-surgeons were correct in $42 \%$ of the trials using only vision feedback and $62 \%$ of the trials using only force feedback. Tukey's method calculated values of $\mathrm{T}_{0.05}$ for surgeons and non-surgeons were 0.2095 and 0.2194 respectively. However, the difference in the means between vision feedback and force feedback for surgeons and non-surgeons was 0.10 and 0.20 
respectively. Therefore, we cannot conclude that this hypothesis is true on an individual group basis.

Hypothesis 2: Providing simultaneous vision and force feedback for all three samples is better than only vision feedback or only force feedback for all three samples $\left((V+F)_{a}>V_{a},(V+F)_{a}>F_{a}\right)$.

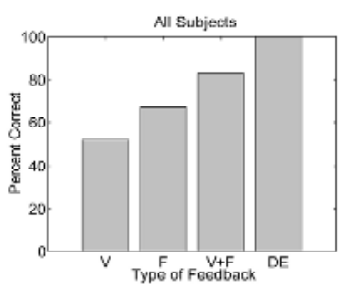

Fig. 7. Percent correct tissue characterizations between the three different artificial tissues using the four methods.

Our second hypothesis states that $(\mathrm{V}+\mathrm{F})_{\mathrm{a}}$ was better than $\mathrm{V}_{\mathrm{a}}$ or $\mathrm{F}_{\mathrm{a}}$ for tissue characterization. As shown in figure 7 , the percent correct for vision, force, and simultaneous vision and force feedback was 52\%, 67\%, and $83 \%$ respectively. A statistical analysis using Tukey's method was performed to determine the significance between the data sets. As explained above, the value of $\mathrm{T}_{0.05}$ was calculated to be 0.1525 . The differences in the means, as shown by the data, between $(\mathrm{V}+\mathrm{F})_{\mathrm{a}}$ and $\mathrm{V}_{\mathrm{a}}$ and also $(\mathrm{V}+\mathrm{F})_{\mathrm{a}}$ and $\mathrm{F}_{\mathrm{a}}$ was 0.31 and 0.16 respectively. Therefore, both means were greater than the $\mathrm{T}_{0.05}$ value signifying a statistical difference between the data sets and validating the hypothesis.

Individually, the surgeons' performance was $62 \%, 72 \%$, and $84 \%$ correct for $\mathrm{V}_{\mathrm{a}}$, $\mathrm{F}_{\mathrm{a}}$, and $(\mathrm{V}+\mathrm{F})_{\mathrm{a}}$ respectively and non-surgeons performance was $42 \%, 62 \%$, and $82 \%$ correct for $\mathrm{V}_{\mathrm{a}}, \mathrm{F}_{\mathrm{a}}$, and $(\mathrm{V}+\mathrm{F})_{\mathrm{a}}$ respectively. Tukey's method was performed and showed calculated $\mathrm{T}_{0.05}$ values of 0.2095 and 0.2194 for surgeons and non-surgeons respectively. Comparing simultaneous vision and force feedback to vision feedback showed the difference in the means for surgeons and non-surgeons of 0.22 and 0.40 , which were both greater than their respective $\mathrm{T}_{0.05}$ value. Therefore, the hypothesis is further supported on an individual group basis. The comparison of simultaneous vision and force feedback to force feedback is not necessary, as a surgical setting will not have only force feedback.

\section{Conclusion}

We have developed our initial prototype of our automated laparoscopic grasper with force feedback and a quick-change tool feature for accommodating cutting and dissection tools at the tool tip. These improvements over conventional tools have made it much easier to use with robotic systems, while still retaining advantages during conventional laparoscopic surgery. The experimental work has demonstrated these advantages by showing that an operator can easily differentiate tissue samples of varying stiffness. Overall, considering all 20 subjects, hypothesis $2\left((\mathrm{~V}+\mathrm{F})_{\mathrm{a}}>\mathrm{V}_{\mathrm{a}}\right.$, $\left.(\mathrm{V}+\mathrm{F})_{\mathrm{a}}>\mathrm{F}_{\mathrm{a}}\right)$ was validated through our experimental results.

While this prototype has achieved our goal of a laparoscopic tool with force feedback, there are issues with our prototype that must be resolved. Future versions of our laparoscopic tool must be scaled down further to incorporate this tool in all commonly used laparoscopic procedures. In addition, we envision using small piezoelectric sensors placed on the jaws to record the forces exerted on the tissue directly instead of the present current-based method. 


\section{References}

1. Chen, H.S., Sheen-Chenn: Synchronous and early metachronous colorectal adenocarcinoma: Analysis of prognosis and current trends. Diseases of the Colon and Rectum, 43 (2000) 1093-1099

2. Bicchi, A., Canepa, G., DeRossi, D., Iacconi, P., Scilingo, E.: A sensor-based minimally invasive surgery tool for detecting tissue elastic properties. IEEE International Conference on Robotics and Automation. 1 (1996) 884-888

3. Hu, T., Castellanos, A.E., Tholey, G., Desai, J.P.: Real-Time Haptic feedback in Laparoscopic tool for use in Gastro-intestinal Surgery. Fifth International Conference on Medical Image Computing and Computer Assisted Intervention (MICCAI), Tokyo, Japan. (2002)

4. Munoz, V.F., Vara-Thorbeck, C., DeGabriel, J.G., Lozano, J.F., Sanchez-Badajoz, E., Garcia-Cerezo, A., Toscano, R., Jimenez-Garrido, A.: A medical robotic assistant for minimally invasive surgery. IEEE International Conference on Robotics and Automation. 3 (2000) 2901-2906

5. Scilingo, E., DeRossi, D., Bicchi, A., Iacconi, P.: Sensor and devices to enhance the performance of a minimally invasive surgery tool for replicating surgeon's haptic perception of the manipulated tissues. IEEE International Conference on Engineering in Medicine and Biology. 3 (1997) 961-964

6. Dingshoft, V.V.H. tot, Lazeroms, M., van der Ham, A., Jongkind, W., Hondred, G.: Force reflection for a laparoscopic forceps. 18th Annual Intenational Conference of the IEEE Engineering in Medicine and Biology Society. 1 (1996) 210-211

7. Rosen, J., Hannaford, B., MacFarlane, M.P., Sinanan, M.N.: Force controlled and teleoperated endoscopic grasper for minimally invasive surgery - experimental performance evaluation. IEEE Transactions on Biomedical Engineering, 46(10). (1999) 1212-1221.

8. Tholey, G., Chanthasopeephan, T., Hu, T., Desai, J.P., Lau, A.: Measuring Grasping and Cutting Forces for Reality-Based Haptic Modeling. Computer Assisted Radiology and Surgery, London, UK. (2003)

9. Dario, P., Bergamasco, M.: An advanced robot system for automated diagnostic task through palpation. IEEE Transactions on Biomedical Engineering, 35 (1998) 118-126.

10. Menciassi, A., Eisinberg, A., Scalari, G., Anticoli, C., Carroza, M., Dario P.: Force feedback-based microinstruments for measuring tissue properties and pulse in microsurgery. IEEE International Conference on Robotics and Automation. 1 (2001) 626-631 